\title{
Urinary biomarkers predict advanced acute kidney injury after cardiovascular surgery
}

\author{
Jian-Jhong Wang ${ }^{1,2}$, Nai-Hsin Chi ${ }^{3}$, Tao-Min Huang ${ }^{2,4}$, Rory Connolly ${ }^{5}$, Liang Wen Chen ${ }^{3}$, Shih-Chieh Jeff Chueh ${ }^{6}$,
} Wei-Chih Kan ${ }^{7}$, Chih-Cheng Lai ${ }^{8}$, Vin-Cent Wu ${ }^{2,4^{*}}$, Ji-Tseng Fang ${ }^{9,10}$, Tzong-Shinn Chu ${ }^{2,4}$ and Kwan-Dun Wu ${ }^{2,4}$

\begin{abstract}
Background: Acute kidney injury (AKI) after cardiovascular surgery is a serious complication. Little is known about the ability of novel biomarkers in combination with clinical risk scores for prediction of advanced AKl.

Methods: In this prospectively conducted multicenter study, urine samples were collected from 149 adults at 0, 3, 6, 12 and $24 \mathrm{~h}$ after cardiovascular surgery. We measured urinary hemojuvelin (uHJV), kidney injury molecule-1 (UKIM-1), neutrophil gelatinase-associated lipocalin (UNGAL), a-glutathione S-transferase (ua-GST) and $\pi$-glutathione S-transferase (UT-GST). The primary outcome was advanced AKI, under the definition of Kidney Disease: Improving Global Outcomes (KDIGO) stage 2, 3 and composite outcomes were KDIGO stage 2, 3 or 90-day mortality after hospital discharge.

Results: Patients with advanced AKI had significantly higher levels of uHJV and UKIM-1 at 3, 6 and $12 \mathrm{~h}$ after surgery. When normalized by urinary creatinine level, UKIM-1 in combination with uHJV at $3 \mathrm{~h}$ post-surgery had a high predictive ability for advanced AKI and composite outcome ( $A \cup C=0.898$ and 0.905 , respectively). The combination of this biomarker panel (normalized UKIM-1, uHJV at $3 \mathrm{~h}$ post-operation) and Liano's score was superior in predicting advanced AKI (AUC $=0.931$, category-free net reclassification improvement of 1.149 , and $p<0.001$ ).
\end{abstract}

Conclusions: When added to Liano's score, normalized UHJV and UKIM-1 levels at $3 \mathrm{~h}$ after cardiovascular surgery enhanced the identification of patients at higher risk of progression to advanced AKI and composite outcomes.

Keywords: Biomarkers, Acute kidney injury, Hemojuvelin, Kidney injury molecule-1, Neutrophil gelatinase-associated lipocalin, a-Glutathione S-transferase, $\pi$-Glutathione S-transferase, Liano's score

\section{Background}

Patients with acute kidney injury (AKI) have increased mortality, prolonged hospital stay and accelerated progression to chronic kidney disease (CKD) [1]. AKI is associated with high morbidity and mortality despite advances in modern medical care [2, 3]. Early and exact detection of potentially significant AKI is important in clinical practice and could lead to timely management [4]. Serum creatinine has traditionally served as a surrogate of renal function, despite its limitations as a diagnostic predictor of AKI [5]. The limitations of serum creatinine include a lack of steady-state conditions in

\footnotetext{
* Correspondence: q91421028@ntu.edu.tw

${ }^{2}$ NSARF group (National Taiwan University Hospital Study Group of ARF),

Taipei, Taiwan

${ }^{4}$ Division of Nephrology, Department of Internal Medicine, National Taiwan

University Hospital, Taipei, Taiwan

Full list of author information is available at the end of the article
}

critically ill patients, with the determinants of serum creatinine (rate of production, apparent volume of distribution and rate of elimination) being variable in the ICU setting.

Novel biomarkers can detect renal tubular injury earlier than serum creatinine in the setting of AKI [6-8]. As these markers correlate with renal tubular injury or function, their concentrations in urine over time, alone or in combination, could provide important information on the progression of AKI [9]. However, most of those patients who develop AKI experience a milder form of AKI (e.g., Kidney Disease: Improving Global Outcomes (KDIGO) stage 1) with transient shifts in serum creatinine, and do not progress to more advanced stages of AKI (KDIGO stage 2 or 3) or require acute dialysis [1012]. Whereas previous studies have attempted to predict AKI (defined as worsening of KDIGO stage) rather than

(C) The Author(s). 2018 Open Access This article is distributed under the terms of the Creative Commons Attribution 4.0 International License (http://creativecommons.org/licenses/by/4.0/), which permits unrestricted use, distribution, and reproduction in any medium, provided you give appropriate credit to the original author(s) and the source, provide a link to the Creative Commons license, and indicate if changes were made. The Creative Commons Public Domain Dedication waiver (http://creativecommons.org/publicdomain/zero/1.0/) applies to the data made available in this article, unless otherwise stated. 
immediate assessment after potential kidney insult, a panel of biomarkers could be used to predict progression of AKI to advanced AKI or mortality among patients after kidney injury (e.g., cardiovascular surgery) $[10,13,14]$. Therefore, we validated three urinary markers extensively upregulated in renal proximal tubules in response to ischemia-reperfusion AKI: urinary hemojuvelin (uHJV) [8], urinary kidney injury molecule1 (uKIM-1) [15] and urinary $\alpha$-glutathione S-transferase (uo-GST) [16], and one marker of distal tubule damage, urinary $\pi$-glutathione S-transferase (uл-GST) [16] (see Additional file 1). We further measured urinary neutrophil gelatinase-associated lipocalin (uNGAL), the well-studied and established inflammatory biomarker identified both in proximal and distal renal tubular damage [17], to validate the prediction of AKI following sequential biomarker measurements after cardiovascular surgery.

Moreover, multiple AKI severity scores have also been derived to predict patient outcome [18]. We further compared the contribution of the clinical models of Liano's score (see Additional file 1) [19], Cleveland Clinic acute renal failure score [20], and Sequential Organ Failure Assessment (SOFA) score [21], and combined these with the predictive power of the urinary biomarkers in an effort to identify and validate the best prediction model for advanced AKI and composite outcomes in patients undergoing cardiovascular surgery.

We hypothesized that some combinations of clinical scores and these site-orientated renal biomarkers would provide better diagnostic accuracy than either alone. In addition, these combinations might involve biomarkers that reflect differing aspects of the pathogenesis of advanced $\mathrm{AKI}$ in this population.

\section{Methods}

\section{Study population}

This study was conducted by the biomarker investigation group from the National Taiwan University Study Group on Acute Renal Failure (NSARF) (see Additional file 1), using a multicenter, prospectively constructed database of AKI [22-26]. Patients undergoing cardiovascular surgery (including coronary bypass, valvular operations and aortic aneurysm repair) between August 2009 and December 2014, were enrolled prospectively from a tertiary center in northern Taiwan and two regional hospitals in central and northern Taiwan. Patients with the following conditions were excluded: those who were younger than 18 years of age, those who were diagnosed with AKI under the KDIGO definitions during index hospitalization before surgery, those who had undergone renal replacement therapy, those who had a history of nephrectomy or renal transplantation and estimated glomerular filtration rate $(\mathrm{eGFR})<30 \mathrm{~mL} / 1.73 \mathrm{~m}^{2}$ at the time of ICU enrollment.
The enrollee was required to have a baseline serum creatinine measurement, defined as 7-180 days prior to the index hospital admission.

\section{Ethics and consent}

This study was approved by the research ethics review board of National Taiwan University Hospital (201105040RC) along with established written informed consent. This research was carried out in accordance with the approved guidelines. Written informed consent was obtained from all participants before inclusion. This study was conducted in accordance with the Declaration of Helsinki.

\section{Clinical data collection}

Medical records of study participants were prospectively reviewed to retrieve hospitalization data, including baseline demographic characteristics (Table 1), intervention procedures, and comorbidity status. Creatinine level with the accompanying eGFR (through the Modification of Diet in Renal Disease (MDRD) Study equation formula) was used for eGFR in this study. Among adults, the MDRD Study equation provides a clinically useful estimate of GFR in patients with stable kidney status [27]; urine output was recorded at each time point after surgery as detailed in the study protocol. Postoperative inotropic agent use was quantified as per inotropic equivalents (see Additional file 1) [23, 25, 28]. Surgeryrelated parameters included surgical methods, aortic clamping time and cardiopulmonary bypass time. Lengths of total admission and ICU admission were also recorded. Disease severity was evaluated as post-surgical SOFA score. The clinical classifications of Liano's score [19] and Cleveland Clinic Foundation Acute Renal Failure Scoring System (CCF ARF score) [20] were also assessed to examine the risk of postoperative renal failure. Clinical and demographic characteristics of these patients were collected at each site on a case report form, which was sent to the coordinating center for entry into the NSARF database [29-31].

\section{Sample collection}

Fresh urine samples were obtained in the ICU at $0,3,6$, 12 and $24 \mathrm{~h}$ after completion of surgery. Other laboratory examinations were performed as indicated clinically. Urine specimens were collected by a standardized procedure, centrifuged within $1 \mathrm{~h}$ and the sediments discarded. The urine samples, collected in separate polypropylene tubes containing sodium azide, were stored at $-80{ }^{\circ} \mathrm{C}$ until required. Each specimen was centrifuged ( $800 \mathrm{~g}$ at $4{ }^{\circ} \mathrm{C}$ for $5 \mathrm{~min}$ ) and the supernatant was collected for ELISA. 
Table 1 Summary of baseline and clinical characteristics of the study patients

\begin{tabular}{|c|c|c|c|c|}
\hline & All & No AKI or stage $1 \mathrm{AKI}$ & Stage 2 or $3 \mathrm{AKI}$ & $p$ value \\
\hline & $(n=149)$ & $(n=131)$ & $(n=18)$ & \\
\hline \multicolumn{5}{|l|}{ Patient characteristics } \\
\hline Age & $62.36 \pm 13.64$ & $62.32 \pm 13.62$ & $62.72 \pm 14.23$ & 0.907 \\
\hline Gender (male) & $104(69.8 \%)$ & $95(72.5 \%)$ & $9(50.0 \%)$ & 0.051 \\
\hline BMl & $24.87 \pm 3.73$ & $24.82 \pm 3.48$ & $25.26 \pm 5.33$ & 0.735 \\
\hline \multicolumn{5}{|l|}{ Comorbidities } \\
\hline Hypertension & $77(51.7 \%)$ & $69(52.7 \%)$ & $8(44.4 \%)$ & 0.512 \\
\hline Diabetes mellitus & $36(24.2 \%)$ & $32(24.4 \%)$ & $4(22.2 \%)$ & 0.838 \\
\hline COPD & $4(2.7 \%)$ & $4(3.1 \%)$ & $0(0.0 \%)$ & 0.452 \\
\hline Liver cirrhosis & $4(2.7 \%)$ & $4(3.1 \%)$ & $0(0.0 \%)$ & 0.452 \\
\hline Congestive heart failure & $14(9.4 \%)$ & $14(10.7 \%)$ & $0(0.0 \%)$ & 0.145 \\
\hline Malignancy & $5(3.4 \%)$ & $4(3.1 \%)$ & $1(5.6 \%)$ & 0.58 \\
\hline \multicolumn{5}{|l|}{ Laboratory data at admission } \\
\hline Preoperative creatinine (mg/dL) & $1.18 \pm 0.33$ & $1.17 \pm 0.31$ & $1.26 \pm 0.43$ & 0.388 \\
\hline eGFR (MDRD) (mL/min/1.73 m²) & $63.27 \pm 20.59$ & $63.86 \pm 20.29$ & $58.97 \pm 22.85$ & 0.346 \\
\hline eGFR between 30 and $60 \mathrm{~mL} / \mathrm{min} / 1.73 \mathrm{~m}^{2}$ & $63(42.3 \%)$ & $56(42.7 \%)$ & $7(38.9 \%)$ & 0.756 \\
\hline Hemoglobin (g/dL) & $13.02 \pm 2.02$ & $13.18 \pm 1.96$ & $11.83 \pm 2.17$ & $0.007^{*}$ \\
\hline Albumin (g/dL) & $4.15 \pm 0.63$ & $4.26 \pm 0.47$ & $3.40 \pm 1.03$ & $0.003^{*}$ \\
\hline LVEF $<55 \%$ & 35 (23.5\%) & $31(23.7 \%)$ & $4(22.2 \%)$ & 0.892 \\
\hline LVEF $<35 \%$ & $10(6.7 \%)$ & $9(6.9 \%)$ & $1(5.6 \%)$ & 0.834 \\
\hline \multicolumn{5}{|l|}{ Perioperative condition } \\
\hline Inotropic equivalents & $5.83 \pm 6.61$ & $4.82 \pm 4.45$ & $13.10 \pm 12.81$ & $0.014^{*}$ \\
\hline Presence of CPB & $93(62.4 \%)$ & $81(61.8 \%)$ & $12(66.7 \%)$ & 0.691 \\
\hline CPB time (min) & $106.57 \pm 98.87$ & $102.23 \pm 97.43$ & $143.27 \pm 111.96$ & 0.200 \\
\hline Presence of cross clamp & $72(48.3 \%)$ & $63(48.1 \%)$ & $9(50.0 \%)$ & 0.879 \\
\hline Clamp time (min) & $57.41 \pm 65.11$ & $55.66 \pm 65.18$ & $78.20 \pm 63.82$ & 0.295 \\
\hline \multicolumn{5}{|l|}{ Operative method } \\
\hline CABG & $77(51.7 \%)$ & $70(53.4 \%)$ & 7 (38.9\%) & 0.247 \\
\hline Valve & $64(43.0 \%)$ & $55(42.0 \%)$ & $9(50.0 \%)$ & 0.519 \\
\hline Aorta & $20(13.4 \%)$ & $16(12.2 \%)$ & $4(22.2 \%)$ & 0.243 \\
\hline \multicolumn{5}{|l|}{ Post-surgery } \\
\hline SOFA score & $6.14 \pm 2.96$ & $5.84 \pm 2.69$ & $8.47 \pm 3.92$ & $0.015^{*}$ \\
\hline Respiratory & $0.67 \pm 0.75$ & $0.66 \pm 0.73$ & $0.77 \pm 1.01$ & 0.608 \\
\hline Coagulation & $0.43 \pm 0.60$ & $0.40 \pm 0.57$ & $0.69 \pm 0.85$ & 0.093 \\
\hline Liver & $0.87 \pm 0.65$ & $0.85 \pm 0.64$ & $1.00 \pm 0.71$ & 0.432 \\
\hline Cardiovascular & $0.28 \pm 0.55$ & $0.24 \pm 0.48$ & $0.62 \pm 0.96$ & 0.191 \\
\hline Central nervous system & $3.08 \pm 1.20$ & $3.08 \pm 1.21$ & $3.08 \pm 1.12$ & 0.997 \\
\hline Renal function & $0.72 \pm 0.79$ & $0.58 \pm 0.66$ & $2.07 \pm 0.64$ & $<0.001^{*}$ \\
\hline Cleveland score & $3.80 \pm 1.62$ & $3.79 \pm 1.56$ & $3.89 \pm 2.03$ & 0.802 \\
\hline Liano's score & $-0.60 \pm 0.84$ & $-0.61 \pm 0.84$ & $-0.47 \pm 0.87$ & 0.506 \\
\hline Length of admission (days) & $18.69 \pm 37.62$ & $17.56 \pm 38.51$ & $30.75 \pm 24.41$ & 0.247 \\
\hline Length of ICU admission (days) & $3.55 \pm 3.30$ & $3.43 \pm 2.84$ & $7.64 \pm 5.35$ & $0.027^{*}$ \\
\hline 90-day mortality & $16(10.7 \%)$ & $6(4.6 \%)$ & $10(55.6 \%)$ & $<0.001^{*}$ \\
\hline
\end{tabular}

Values are mean \pm SD or number (percentage)

$A K I$ acute kidney injury, $B M I$ body mass index, $C A B G$ coronary artery bypass graft, COPD chronic obstructive pulmonary disease, $C P B$ cardiopulmonary bypass, eGFR estimated glomerular filtration rate, ICU intensive care unit, LVEF left ventricular ejection fraction, MDRD Modification of Diet in Renal Disease Study equation, SOFA score Sequential Organ Failure Assessment score

${ }^{*} p<0.05$ 


\section{Biomarker Measurements}

The urinary HJV, KIM-1 and NGAL levels were measured by a human hemojuvelin ELISA kit (USCN Life Science, Inc., Wuhan, China), human urinary TIM-1/ KIM-1/HAVCR Quantikine ELISA kit (R\&D Systems, USA) and a human lipocalin-2/NGAL ELISA kit (R\&D Systems), respectively. All of the results were expressed in nanograms per milliliter. The lower limit of detection for HJV, KIM-1 and NGAL was 0.156, 0.046 and $0.2 \mathrm{ng} /$ $\mathrm{mL}$, respectively. The urinary $\alpha-\mathrm{GST}$ and $\pi-\mathrm{GST}$ levels were determined using Human Alpha and Pi GST EIA Test Kits (EKF Diagnostics). The results were expressed in micrograms per liter. The inter-assay and intra-assay coefficient of variation for $\alpha$-GST was $6.3 \%$ and $2.7 \%$, respectively, and for $\pi$-GST, it was $8.6 \%$ and $3.1 \%$, respectively. The lower limit of detection for $\alpha$-GST and for $\pi-G S T$ was $0.3 \mu \mathrm{g} / \mathrm{L}$. Assays were completed as described by the manufacturer's protocol, and each measurement was performed in duplicate. Urinary creatinine levels were measured using the Jaffe assay, with standardization to isotope dilution mass spectrometry (IDMS)-traceable reference. Technicians performing the biomarker measurements were blinded to each patient's clinical information. All biomarkers were measured from frozen aliquots that did not undergo any additional freeze-thaw cycles.

\section{Outcome definitions}

The clinical primary endpoint was defined as the development of advanced AKI (stage 2 or 3, specified by the KDIGO criteria [32]), with both urine and creatinine criteria applied. The secondary outcomes were 90 -day mortality after hospital discharge and composite outcomes defined as 90-day mortality after hospital discharge or advanced AKI, whichever occurred earlier.

\section{Statistical analysis}

All analyses were performed using SPSS software, version 20 (IBM, Armonk, NY, USA), R software, version 3.2.2 (Free Software Foundation, Inc., Boston, MA, USA), and MedCalc Statistical Software, version 15.11.3 (MedCalc Software bvba, Ostend, Belgium; https://www.medcalc. org; 2015). The two-sample $t$ test or Mann-Whitney rank sum test was used as appropriate to compare continuous variables; for categorical variables, the chi-square $\left(x^{2}\right)$ or Fisher's exact test was applied. Friedman two-way analysis of variance (ANOVA) was used to assess the overall difference in HJV, KIM-1, NGAL, $\alpha$-GST and $\pi$-GST between the "no AKI or stage 1 AKI" and the "stage 2 or 3 AKI" groups at $0,3,6,12$ and $24 \mathrm{~h}$ after cardiovascular surgery [33]. We normalized biomarker levels with urine creatinine concentrations and analyzed them at each time point [34]. We fitted logistic regression models to examine the association between each marker (urinary NGAL, HJV,
KIM-1, $\alpha$-GST and $\pi-G S T$ ) and advanced AKI, and generated an area under the receiver-operating characteristic (ROC) curve to assess the predictive accuracy of each marker. Power analysis was conducted based on the prior knowledge that the ratio of cases to controls was 4:1. We required 105 patients (21 with severe AKI and 84 without severe AKI) to achieve power of 0.8 , with type I error of 0 . 05 . This was based on the preliminary knowledge that the discriminatory power of urinary HJV to predict severe AKI was 0.7 [35]. We used a generalized additive model (GAM) (with spline) incorporating the subject-specific (longitudinal) random effects and adjusted for other clinical parameters to predict the outcomes [36, 37]. Simple and multiple generalized additive models (GAMs) were fitted to detect nonlinear effects of continuous covariates and identify appropriate cutoff point(s) for discretizing continuous covariates, if necessary, during the stepwise variable selection procedure. We defined the optimal cutoff value as odd equals to zero [38].

To assess the additive prediction ability of each biomarker compared with traditional clinical predictors, we identified clinical risk prediction models (including Liano's score, CCF ARF score and SOFA score) that were based on area under the curve (AUC) analysis and then added each biomarker individually to this clinical model. We estimated the risk score function using a logistic regression model including clinical and biomarker variables as covariates. The Hosmer-Lemeshow logistic regression model test for goodness of fit was used to assess the calibration between the current model and the expected model. Finally, we calculated the net reclassification improvement (NRI) and integrated discrimination improvement (IDI) to estimate overall improvement in reclassification with urinary biomarkers adding to clinical variables [39]. We reclassified the patients who developed advanced AKI using 0-12\%, 12$30 \%$ and $>30 \%$ for the risk categories. A $p$ value $<0.05$ was considered significant.

Hierarchical clustering analysis was performed using the cluster program and the results were visualized using the Treeview program [40]. The biomarkers were arranged in such a way that the most similar expression profiles were placed next to each other. In the color scheme, strong positive staining is indicated as a red cube, weak positive staining as a black cube and negative staining as a green cube. Absence of staining data is indicated with a grey cube.

\section{Results}

\section{Clinical characteristics}

A total of 149 patients who underwent cardiovascular surgery were enrolled in this study, with subsequent time-varying sample collection. There were 46 patients who had AKI episodes as defined by KDIGO criteria (30. 
9\%), with 28 (18.8\%) having stage 1 AKI, 11 (7.4\%) stage 2 AKI, and 7 (4.7\%) progressing to stage 3 AKI: 6 patients progressed to dialysis-dependent AKI in the advanced AKI group. The 90-day mortality rate in this cohort was $10.7 \%$. The clinical characteristics of patients with and without advanced AKI are described in Table 1. Patients who developed advanced AKI had lower hemoglobin and albumin levels, were administered higher inotropic equivalents and had higher SOFA scores. However, there were no statistical differences between patients with and without advanced AKI with respect to age, gender, body mass index (BMI), comorbidities, baseline kidney function, baseline chronic kidney disease (eGFR between 30 and $60 \mathrm{ml} / \mathrm{min} / 1.73 \mathrm{~m}^{2}$ ) status and the Liano and CCF ARF disease severity scores. Clinical outcomes were worse for patients in the advanced AKI group when they had longer ICU stays and a higher 90-day mortality rate, even after discharge.

\section{Relationship between biomarker levels and advanced AKI}

We compared urinary biomarker levels between patients with and without advanced AKI (patients without AKI or patients with stage $1 \mathrm{AKI}$ ): the normalized urine biomarker data were transformed logarithmically to avoid the interference of numbers in the extreme and to approximate normal distribution in the analyses (Fig. 1). At $3 \mathrm{~h}$ post-surgery, normalized uHJV $(p=0.006)$, and uKIM-1 $(p=0.019)$ levels in the patients with advanced

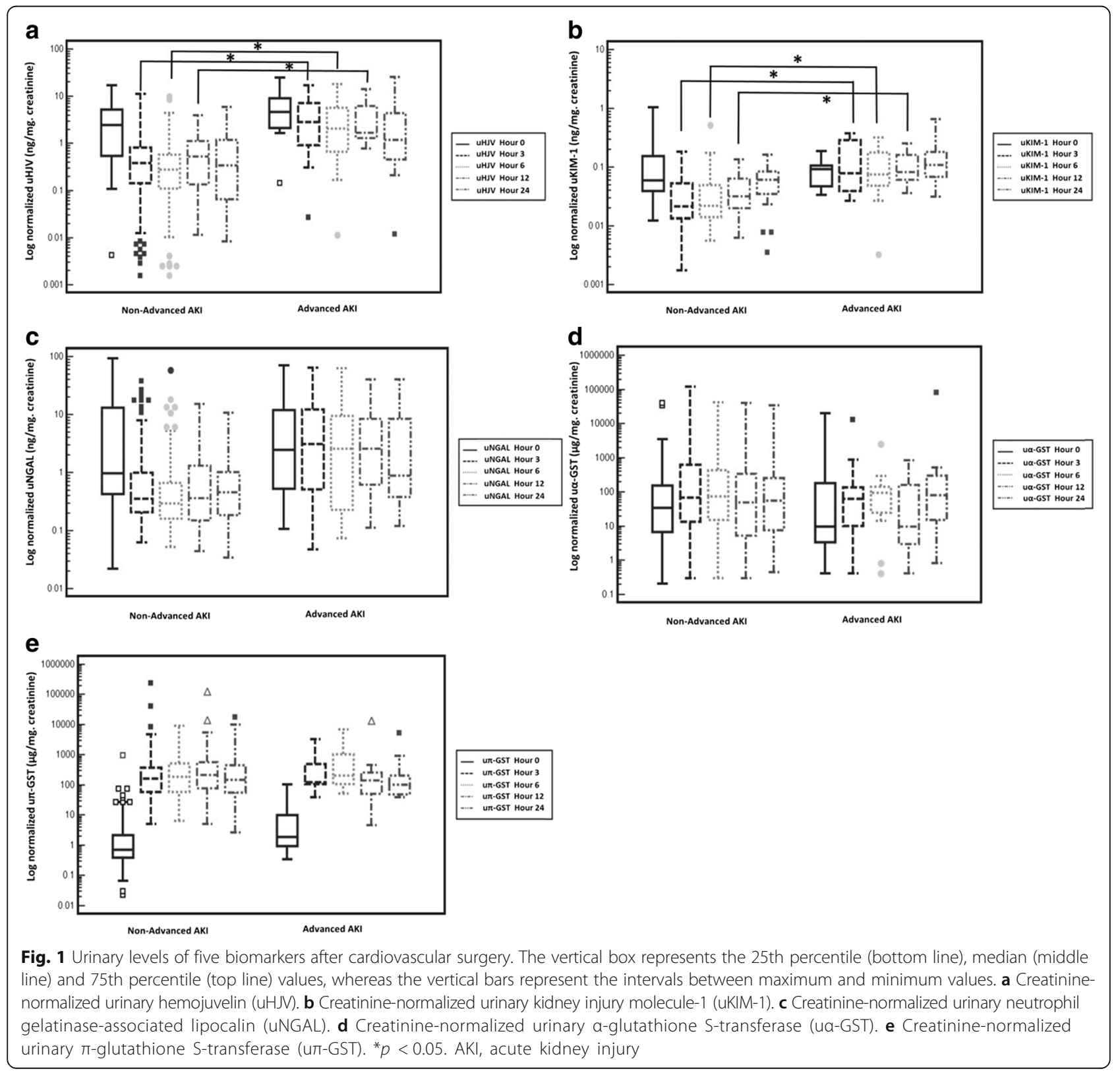


AKI were significantly higher than those without advanced AKI. Moreover, considerably higher levels of uHJV and uKIM-1 were observed at 6 and $12 \mathrm{~h}$ postsurgery. However, there were no significant differences

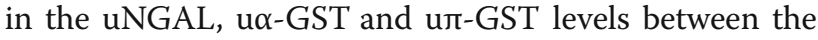
patients with and without advanced AKI at these sequential time points. All biomarkers analyzed by Friedman two-way ANOVA (HJV, KIM-1, NGAL, $\alpha$-GST and $\pi$-GST) were significantly different (all $p<0.05$ ) between the no AKI/stage $1 \mathrm{AKI}$ and the stage $2 / 3 \mathrm{AKI}$ groups at each of the time points of $0,3,6,12$ and $24 \mathrm{~h}$ after cardiovascular surgery.

\section{Urinary biomarkers and the prediction of advanced AKI}

Among the patients who subsequently developed advanced AKI, the, uHJV biomarker had the highest AUC values, particularly at $12 \mathrm{~h}$ post-surgery. Following normalization with urinary creatinine concentration, the predictive ability of several urinary biomarkers was improved, especially normalized uHJV at $12 \mathrm{~h}$ post-surgery (Table 2). When compared to serum creatinine, normalized uHJV and uKIM-1 had better performance for predicting advanced AKI at 3 and $6 \mathrm{~h}$ post-surgery (Additional file 1: Table S1 and Fig. 1). The combined panel of normalized uHJV and uKIM-1 at $3 \mathrm{~h}$ post- surgery had the largest AUC (AUC $=0.898,95 \%$ CI 0. 80-0.96) compared to any of the other two or three biomarker combinations (Table 3 and Fig. 2). This combination had a positive predictive value of $42 \%$, negative predictive value of $100 \%$, sensitivity of $100 \%$ and specificity of $70 \%$. The GAM plot was generated thereafter and demonstrated positive correlation between increased normalized $\mathrm{uHJV}$ and $\mathrm{uKIM}-1$ at $3 \mathrm{~h}$ post-surgery and the risk of developing advanced AKI. The cutoff value 2 . $346 \mathrm{ng} / \mathrm{mL}$ for normalized $\mathrm{uHJV}$ and $0.047 \mathrm{ng} / \mathrm{mL}$ for normalized uKIM-1 performed best to predict advanced AKI (Additional file 1: Figure S2).

\section{Adding urinary biomarkers to clinical risk prediction models}

In combining the predictive ability of novel biomarkers and various clinical scoring systems for AKI, we found that the greatest AUC was observed with the combination of Liano's AKI score and the panel of normalized uHJV and uKIM-1 at $3 \mathrm{~h}$ after surgery, with an AUC of 0.931 to predict advanced AKI. This panel had a greater AUC value than other combinations of biomarkers at 3 , 6, 12, or $24 \mathrm{~h}$ after surgery (Table 4 and Fig. 2).

We assessed reclassification and evaluated any improvement in detection of advanced AKI events. The

Table 2 Area under the receiver-operating characteristic curve at each time point for urinary biomarkers with and without normalization to urinary creatinine for predicting advanced acute kidney injury

\begin{tabular}{|c|c|c|c|c|c|c|c|}
\hline Urinary Biomarkers & Time after enrollment & AUC & $95 \% \mathrm{Cl}$ & Urinary biomarkers & Time after enrollment & AUC & $95 \% \mathrm{Cl}$ \\
\hline \multirow[t]{4}{*}{ uHJV } & Hour 3 & 0.793 & 0.709 to 0.862 & Normalized uHJV & Hour 3 & 0.833 & 0.753 to 0.895 \\
\hline & Hour 6 & 0.802 & 0.720 to 0.869 & & Hour 6 & 0.808 & 0.726 to 0.874 \\
\hline & Hour 12 & 0.813 & 0.683 to 0.907 & & Hour 12 & 0.841 & 0.715 to 0.927 \\
\hline & Hour 24 & 0.687 & 0.542 to 0.809 & & Hour 24 & 0.719 & 0.575 to 0.835 \\
\hline \multirow[t]{4}{*}{ uKIM-1 } & Hour 3 & 0.670 & 0.549 to 0.776 & Normalized uKIM-1 & Hour 3 & 0.819 & 0.710 to 0.900 \\
\hline & Hour 6 & 0.664 & 0.543 to 0.771 & & Hour 6 & 0.787 & 0.675 to 0.875 \\
\hline & Hour 12 & 0.602 & 0.451 to 0.741 & & Hour 12 & 0.831 & 0.695 to 0.923 \\
\hline & Hour 24 & 0.544 & 0.391 to 0.692 & & Hour 24 & 0.772 & 0.625 to 0.882 \\
\hline \multirow[t]{4}{*}{ UNGAL } & Hour 3 & 0.711 & 0.621 to 0.790 & Normalized UNGAL & Hour 3 & 0.707 & 0.617 to 0.787 \\
\hline & Hour 6 & 0.754 & 0.667 to 0.828 & & Hour 6 & 0.691 & 0.600 to 0.772 \\
\hline & Hour 12 & 0.660 & 0.517 to 0.785 & & Hour 12 & 0.745 & 0.607 to 0.855 \\
\hline & Hour 24 & 0.640 & 0.493 to 0.769 & & Hour 24 & 0.691 & 0.546 to 0.813 \\
\hline \multirow[t]{4}{*}{ ua-GST } & Hour 3 & 0.504 & 0.419 to 0.589 & Normalized ua-GST & Hour 3 & 0.556 & 0.470 to 0.639 \\
\hline & Hour 6 & 0.523 & 0.437 to 0.608 & & Hour 6 & 0.54 & 0.454 to 0.624 \\
\hline & Hour 12 & 0.573 & 0.487 to 0.656 & & Hour 12 & 0.61 & 0.524 to 0.691 \\
\hline & Hour 24 & 0.633 & 0.547 to 0.713 & & Hour 24 & 0.542 & 0.456 to 0.627 \\
\hline \multirow[t]{4}{*}{ UT-GST } & Hour 3 & 0.669 & 0.584 to 0.745 & Normalized un-GST & Hour 3 & 0.547 & 0.462 to 0.631 \\
\hline & Hour 6 & 0.779 & 0.702 to 0.845 & & Hour 6 & 0.591 & 0.505 to 0.673 \\
\hline & Hour 12 & 0.586 & 0.500 to 0.668 & & Hour 12 & 0.594 & 0.508 to 0.676 \\
\hline & Hour 24 & 0.631 & 0.545 to 0.710 & & Hour 24 & 0.548 & 0.462 to 0.632 \\
\hline
\end{tabular}

AUC area under the receiver-operating characteristic curve, $C$ confidence interval, $u H J V$ urinary hemojuvelin, uKIM-1 urinary kidney injury molecule-1, uNGAL urinary neutrophil gelatinase-associated lipocalin, ua-GST urinary a-glutathione S-transferases, um-GST urinary n-glutathione S-transferases 
Table 3 Logistic regression analysis with variables available for predicting advanced acute kidney injury and model accuracy after combining urinary biomarkers

\begin{tabular}{|c|c|c|}
\hline Model & AUC (95\% Cl) & $p^{b}$ \\
\hline \multicolumn{3}{|l|}{ Hour 3} \\
\hline Normalized uHJV & 0.833 (0.753 to 0.895$)$ & NA \\
\hline Normalized (uHJV + UKIM-1) ${ }^{a, c}$ & 0.898 (0.804 to 0.957$)$ & 0.468 \\
\hline Normalized (uHJV + uNGAL) & 0.831 (0.751 to 0.893$)$ & 0.274 \\
\hline Normalized (uKIM-1 + uNGAL) & 0.862 (0.760 to 0.932 ) & 0.943 \\
\hline Normalized (uHJV + uKIM-1 + uNGAL) & 0.897 (0.803 to 0.956$)$ & 0.496 \\
\hline \multicolumn{3}{|l|}{ Hour 6} \\
\hline Normalized uHJV & 0.808 (0.726 to 0.874$)$ & NA \\
\hline Normalized (uHJV + uKIM-1) & 0.827 (0.719 to 0.906$)$ & 0.785 \\
\hline Normalized (uHJV + uNGAL) & 0.808 (0.726 to 0.874$)$ & 1.00 \\
\hline Normalized (uKIM-1 + uNGAL) & 0.816 (0.707 to 0.898$)$ & 0.712 \\
\hline Normalized (uHJV + uKIM-1 + uNGAL) & 0.834 (0.728 to 0.912$)$ & 0.634 \\
\hline \multicolumn{3}{|l|}{ Hour 12} \\
\hline Normalized uHJV & 0.841 (0.715 to 0.927 ) & NA \\
\hline Normalized (uHJV + uKIM-1) & 0.890 (0.766 to 0.962 ) & 0.332 \\
\hline Normalized (uHJV + uNGAL) & 0.850 (0.725 to 0.933$)$ & 0.706 \\
\hline Normalized (uKIM-1 + uNGAL) & 0.866 (0.736 to 0.947$)$ & 0.819 \\
\hline Normalized (uHJV + uKIM-1 + uNGAL) & 0.892 (0.769 to 0.963$)$ & 0.311 \\
\hline \multicolumn{3}{|l|}{ Hour 24} \\
\hline Normalized uKIM-1 & 0.772 (0.625 to 0.882 ) & NA \\
\hline Normalized (uKIM-1 + uHJV) & 0.848 (0.711 to 0.936$)$ & 0.137 \\
\hline Normalized (uKIM-1 + uNGAL) & 0.826 (0.686 to 0.921$)$ & 0.230 \\
\hline Normalized (uHJV + uNGAL) & 0.730 (0.587 to 0.844$)$ & 0.567 \\
\hline Normalized (uKIM-1 + uHJV + uNGAL) & 0.855 (0.720 to 0.941$)$ & 0.085 \\
\hline
\end{tabular}

$\overline{A U C}$ area under the receiver-operating characteristic curve, $\mathrm{Cl}$ confidence interval, $N A$ not applicable, $u H J V$ urinary hemojuvelin, uKIM-1 urinary kidney injury molecule-1, uNGAL urinary neutrophil gelatinase-associated lipocalin

aHosmer-Lemeshow goodness of fit test: $p=0.541$ for the best prediction model

${ }^{\mathrm{b}} \mathrm{Compared}$ with normalized uHJV at hour 3, hour 6 and hour 12 and normalized uKIM-1 at hour 24

'Best prediction model (greatest AUC)

combination of the creatinine-normalized uHJV and uKIM-1 biomarker panel with the Liano's score led to a significant increase in risk stratification (total NRI $=1$. 149; $p<0.001)$. The majority of this effect came from those with advanced AKI events (NRI event $=0.538 ; p<$ 0.001 ), whereas the NRI non-event was 0.610 (95\% CI, $p$ $<0.001$ ) (NRI reclassification table and proportion was shown in Additional file 1: Table S2). Simultaneously, the total integrated discrimination improvement (IDI) was significant at 0.383 (95\% CI, $0.25-0.52 ; p<0.001)$. The combination of normalized UHJV and uKIM-1 with the Liano's score was also significant for NRI and IDI reclassification in forecasting advanced AKI at 6, 12, and $24 \mathrm{~h}$ post-surgery (Table 5).
Combining clinical risk prediction models and urinary biomarkers for prediction of mortality and composite outcomes

The combined panel of normalized uHJV and uNGAL at $3 \mathrm{~h}$ post-surgery had strong predictive ability for 90-day mortality rate after hospital discharge $(\mathrm{AUC}=0.896)$ and the best predictive ability for composite outcomes (AUC $=0.905)$. The predictive ability for composite outcomes further improved after combination with Liano's score $(\mathrm{AUC}=0.943)$ (Additional file 1: Table S3). Given the potential for collinearity between some of the biomarkers, we performed an unsupervised cluster analysis to determine the relationship of the biomarkers to advanced AKI (Fig. 3). The unsupervised clustering resulted in groupings similar to functional groupings for some of the biomarkers. For instance, uHJV and uKIM-1 in the group associated with proximal tubular dysfunction were clustered in the near group, nearest to "advanced AKI", and may explain their strong predictive ability for advanced AKI. Overall, the correlation within each group was stronger for biomarkers that had better predictive ability.

\section{Discussion}

In this prospective multicenter observational study, we have shown that the combined biomarker panel of creatinine-normalized UHJV and UKIM-1 at $3 \mathrm{~h}$ after cardiovascular surgery had the strongest predictive ability for the development of advanced AKI and composite outcomes. This predictive ability was increased further after combination with Liano's score. The combination of clinical models and biologic biomarkers could better anticipate the future development of advanced AKI in the early postoperative period, suggesting future clinical outcomes.

The combination of uHJV and uKIM-1, both markers of structural kidney injury, provided added value to the traditional clinical AKI score in forecasting the risk of advanced AKI. Consequently, combination approaches could account for different time courses of biomarker release or could reflect different pathophysiological mechanisms [41]. HJV, a glycophosphatidylinositol (GPI)-linked membrane protein, is highly expressed in liver and skeletal muscles. The molecular weight of HJV is $42 \mathrm{kDa}$ for the soluble form (sHJV) [42], and can be passed through glomerular filtration and reabsorbed by the renal tubules [8]. Increased iron content in the kidney and urine is observed in human and animal models of AKI [43], and increased iron load can induce renal tubular cell injury [44]. There is evidence that the increased expression of the hemojuvelin-hepcidinferroportin pathway is an intrinsic response to iron overload conditions during AKI. Therefore, uHJV has the potential to be an early AKI biomarker in response to 

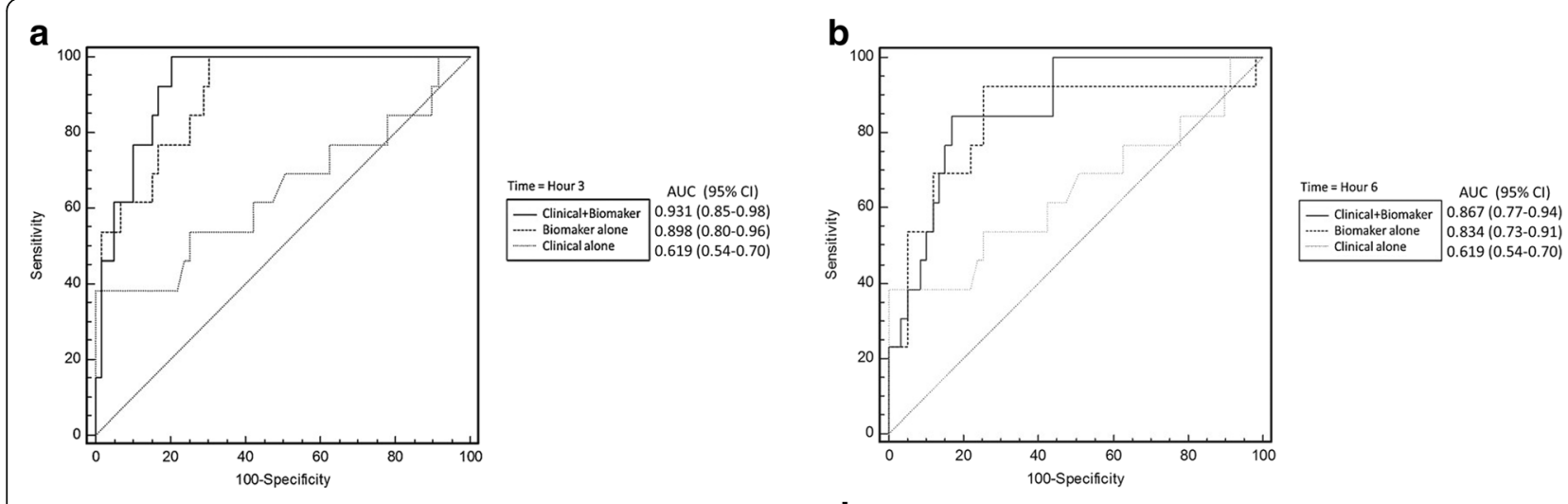

C
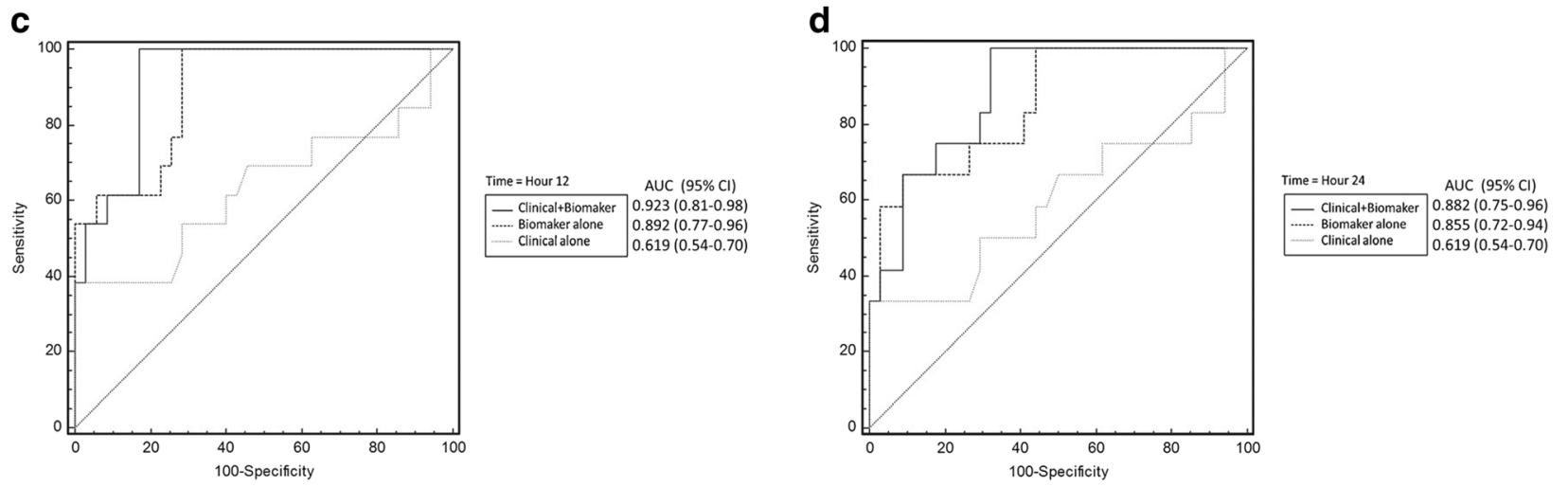

Fig. 2 Receiver-operating characteristic (ROC) curves for the best prediction model for advanced acute kidney injury. The best biomarker panel combination alone (thick dashed line), clinical risk prediction model alone (thin dashed line) and combination of the clinical risk prediction model and combined biomarker panel (solid line) are shown at hour 3 (a), hour $6(\mathbf{b})$, hour 12 (c) and hour 24 (d). The clinical risk prediction model is calculated from Liano's score. The area under the ROC curve (AUC) values and $95 \%$ confidence intervals (Cls) are also shown

iron homeostasis during AKI, which may explain the temporal relationship between uHJV and its predictive capacity. In this study, we showed that the novel biomarker of HJV can predict AKI after cardiac surgery. This finding reinforced our previous finding, in which high urinary HJV levels were observed in patients after cardiac surgery and rhabdomyolysis-related AKI. Although HJV is highly expressed in liver, the post-surgery liver function was not significantly different between the no AKI/stage 1 AKI and the stage 2/3 AKI groups This result suggested higher HJV concentration in patients with advanced AKI was mainly from kidney injury. According to our animal model, the elevation of urinary HJV in acute tubular necrosis should be originally from filtration after renal tubular destruction rather than the liver (see Additional file 1) [8].

Kidney injury molecule-1 (KIM-1), a 38.7-kDa transmembrane protein with immunoglobulin-like and mucin domains in its ectodomain, is a novel biomarker for renal proximal tubule injury and could play a role in tubulo-interstitial damage [45]. In addition to being a biomarker of AKI, KIM-1 could also play a role in renal recovery and tubular regeneration after AKI [46]. However, because increased urinary KIM-1 concentration can indicate either injury or the repair response to injury, KIM-1 by itself may not be a suitable marker for early prediction of AKI whereas the combination of KIM-1 with other injury markers may be highly useful. In support of this hypothesis, our result was consistent with a study investigating biomarker combinations for prediction of AKI following cardiac surgery [14], whereby KIM-1 in combination with another biomarker had the best predictive value for severe AKI.

We further showed that the combination of a panel of biomarkers and Liano's AKI score may be beneficial in providing additional information with respect to the risk of AKI severity and other adverse clinical outcomes. Due to the heterogeneous causes of AKI and underlying comorbidities, the use of biomarkers and clinical disease severity scores to predict renal injury seems reasonable and actually did have better accuracy [17]. For increased accuracy and added predictive ability beyond the AUC value, the biomarker and clinical score combination model was supported by net reclassification improvement (NRI) and integrated discrimination improvement (IDI) values. The NRI and IDI represent modern, more sensitive statistical methods to reclassify model improvement from the use of a biomarker with an existing 
Table 4 Logistic regression analysis with variables available for predicting advanced acute kidney injury and model accuracy after combining clinical models with urinary biomarkers

\begin{tabular}{|c|c|c|}
\hline Model & AUC $(95 \% \mathrm{Cl})$ & $p^{b}$ \\
\hline Liano's score & 0.619 (0.536 to 0.698$)$ & \\
\hline Cleveland score & 0.493 (0.410 to 0.576$)$ & \\
\hline SOFA score & 0.700 (0.619 to 0.773 ) & \\
\hline \multicolumn{3}{|l|}{ Hour 3} \\
\hline Normalized uHJV & 0.833 (0.753 to 0.895$)$ & NA \\
\hline Liano's + normalized uHJV & 0.817 (0.736 to 0.882$)$ & 0.672 \\
\hline Cleveland + normalized uHJV & 0.808 (0.726 to 0.874$)$ & 0.662 \\
\hline SOFA + normalized uHJV & 0.786 (0.701 to 0.856$)$ & 0.419 \\
\hline Normalized (uHJV + uKIM-1) & 0.898 (0.804 to 0.957$)$ & 0.468 \\
\hline Liano's + normalized (UHJV + UKIM-1) $)^{\mathrm{a}, \mathrm{c}}$ & 0.931 (0.846 to 0.977$)$ & 0.206 \\
\hline Cleveland + normalized (uHJV + uKIM-1) & 0.883 (0.785 to 0.946$)$ & 0.817 \\
\hline SOFA + normalized (uHJV + uKIM-1) & 0.876 (0.776 to 0.942$)$ & 0.811 \\
\hline \multicolumn{3}{|l|}{ Hour 6} \\
\hline Normalized uHJV & 0.808 (0.726 to 0.874$)$ & NA \\
\hline Liano's + normalized uHJV & 0.791 (0.707 to 0.860 ) & 0.653 \\
\hline Cleveland + normalized uHJV & 0.769 (0.683 to 0.841$)$ & 0.544 \\
\hline SOFA + normalized uHJV & 0.759 (0.672 to 0.833 ) & 0.426 \\
\hline Normalized (uHJV + uKIM-1 + uNGAL) & 0.834 (0.728 to 0.912$)$ & 0.634 \\
\hline Liano's + normalized (uHJV + uKIM-1 + uNGAL) & 0.867 (0.766 to 0.935$)$ & 0.334 \\
\hline Cleveland + normalized (UHJV + UKIM-1 + uNGAL) & 0.817 (0.709 to 0.899$)$ & 0.985 \\
\hline SOFA + normalized (uHJV + UKIM-1 + uNGAL) & 0.812 (0.702 to 0.895$)$ & 0.920 \\
\hline \multicolumn{3}{|l|}{ Hour 12} \\
\hline Normalized uHJV & 0.841 (0.715 to 0.927$)$ & NA \\
\hline Liano's + normalized uHJV & 0.834 (0.707 to 0.922 ) & 0.879 \\
\hline Cleveland + normalized uHJV & 0.892 (0.776 to 0.960$)$ & 0.429 \\
\hline SOFA + normalized uHJV & 0.834 (0.705 to 0.923$)$ & 0.656 \\
\hline Normalized (uHJV + uKIM-1 + uNGAL) & 0.892 (0.769 to 0.963 ) & 0.311 \\
\hline Liano's + normalized (uHJV + uKIM-1 + uNGAL) & 0.923 (0.808 to 0.980$)$ & 0.151 \\
\hline Cleveland + normalized (UHJV + UKIM-1 + uNGAL) & 0.916 (0.800 to 0.976$)$ & 0.281 \\
\hline SOFA + normalized (uHJV + UKIM-1 + uNGAL) & 0.914 (0.795 to 0.975$)$ & 0.302 \\
\hline \multicolumn{3}{|l|}{ Hour 24} \\
\hline Normalized uKIM-1 & 0.772 (0.625 to 0.882$)$ & NA \\
\hline Liano's + normalized uKIM-1 & 0.799 (0.655 to 0.902$)$ & 0.488 \\
\hline Cleveland + normalized uKIM-1 & 0.765 (0.617 to 0.877$)$ & 0.737 \\
\hline SOFA + normalized uKIM-1 & 0.797 (0.650 to 0.902 ) & 0.587 \\
\hline Normalized (uKIM-1 + uHJV + uNGAL) & 0.855 (0.720 to 0.941$)$ & 0.085 \\
\hline Liano's + normalized (uKIM-1 + uHJV + UNGAL) & 0.882 (0.753 to 0.958$)$ & 0.030 \\
\hline Cleveland + normalized (uKIM-1 + uHJV + uNGAL) & 0.863 (0.729 to 0.946$)$ & 0.096 \\
\hline SOFA + normalized (UKIM-1 + uHJV + uNGAL) & 0.869 (0.735 to 0.951$)$ & 0.082 \\
\hline
\end{tabular}

AUC area under the receiver-operating characteristic curve, $\mathrm{Cl}$ confidence interval, SOFA score Sequential Organ Failure Assessment score, uHJV urinary hemojuvelin, uKIM-1 urinary kidney injury molecule-1, uNGAL urinary neutrophil gelatinase-associated lipocalin

${ }^{a}$ Hosmer-Lemeshow goodness of fit test: $p=0.481$ for the best prediction model

${ }^{b}$ Compared with normalized uHJV at hour 3, hour 6 and hour 12 and normalized UKIM-1 at hour 24

'Best prediction model (greatest AUC), combined clinical model (Liano's score) with biomarkers 
Table 5 Discriminative improvement of combined biomarkers added to Liano's score for prediction of advanced acute kidney injury

\begin{tabular}{|c|c|c|c|c|c|c|}
\hline Model & AUC $(95 \% \mathrm{Cl})$ & $p^{a}$ & $\mathrm{NRI}^{\mathrm{b}}(95 \% \mathrm{Cl})$ & $p^{c}$ & IDI (95\% Cl) & $p$ \\
\hline \multicolumn{7}{|l|}{ Hour 3} \\
\hline Normalized (uHJV + uKIM-1) & 0.898 (0.804 to 0.957$)$ & NA & & & & \\
\hline Liano's + normalized (uHJV + UKIM-1) ${ }^{d}$ & 0.931 (0.846 to 0.977 ) & 0.330 & 1.149 (0.76 to 1.53$)$ & $<0.001$ & 0.383 (0.25 to 0.52$)$ & $<0.001$ \\
\hline \multicolumn{7}{|l|}{ Hour 6} \\
\hline Normalized (uHJV + uKIM-1 + uNGAL) & 0.834 (0.728 to 0.912$)$ & NA & & & & \\
\hline Liano's + normalized (uHJV + uKIM-1 + uNGAL) & 0.867 (0.766 to 0.935$)$ & 0.499 & $1.030(0.64$ to 1.41$)$ & $<0.001$ & 0.343 (0.21 to 0.48$)$ & $<0.001$ \\
\hline \multicolumn{7}{|l|}{ Hour 12} \\
\hline Normalized (uHJV + uKIM-1 + uNGAL) & 0.892 (0.769 to 0.963 ) & NA & & & & \\
\hline Liano's + normalized (uHJV + uKIM-1 + uNGAL) & 0.923 (0.808 to 0.980$)$ & 0.344 & 0.831 (0.40 to 1.27$)$ & $<0.001$ & 0.353 (0.19 to 0.52 ) & $<0.001$ \\
\hline \multicolumn{7}{|l|}{ Hour 24} \\
\hline Normalized (uKIM-1 + uHJV + uNGAL) & 0.855 (0.720 to 0.941$)$ & NA & & & & \\
\hline Liano's + normalized (uKIM-1 + uHJV + UNGAL) & 0.882 (0.753 to 0.958 ) & 0.288 & 1.162 (0.81 to 1.52 ) & $<0.001$ & 0.387 (0.27 to 0.51$)$ & $<0.001$ \\
\hline
\end{tabular}

$A U C$ area under the receiver-operating characteristic curve, $\mathrm{Cl}$ confidence interval, IDI integrated discrimination improvement, $N R I$ net reclassification improvement, UHJV urinary hemojuvelin, uKIM-1 urinary kidney injury molecule-1, uNGAL urinary neutrophil gelatinase-associated lipocalin

${ }^{a}$ Compared with normalized (uHJV + uKIM-1) at hour 3, normalized (uHJV + uKIM-1 + uNGAL) at hour 6, normalized (uHJV + uKIM-1 + uNGAL) at hour 12 and normalized (uKIM-1 + UHJV + uNGAL) at hour 24

${ }^{\mathrm{b}}$ The ability of a risk marker to more accurately stratify individuals into higher or lower risk categories was investigated by NRI. We reclassified the patients who had subsequent advanced acute kidney injury (AKI) or who did not by using a priori risk categories of $0-12 \%, 12-30 \%$ and $>30 \%$ for the risk of advanced AKI 'The $p$ value for increase in NRI in a model with urinary biomarkers combined with Liano's score compared with urinary biomarkers alone

${ }^{\mathrm{d} B e s t}$ prediction model (greatest AUC), combined clinical model (Liano's score) with biomarkers

clinical model. Urinary HJV and uKIM-1 had the unique ability to improve the NRI for both events and nonevents and augmented the AUC value compared with use of a clinical model alone and thus, uHJV and uKIM1 may serve as an ideal biomarker panel to help early detection of advanced AKI.

Biomarkers predicting advanced AKI will allow future clinical trials in AKI intervention to be targeted to those patients at highest risk of disease progression, which would be far more efficient in terms of costs and resources [47]. The majority of patients $(28 / 46,60.9 \%$ in this study) who developed AKI after cardiovascular surgery had stage 1 AKI and this spontaneously resolved. The ability to distinguish those patients at highest risk of severe outcomes will be crucial as nephrologists and intensivists conduct more clinical trials in the treatment of AKI [48]. Given the clinical uncertainty and high complexity of medical decision-making around this issue (e.g., timing and duration of renal replacement therapy, placement of permanent access and follow up), any method that provides a modest improvement in clinical prediction could have clinical importance. We endeavor to find the biomarkers and clinical severity score to determine which patients will develop advanced AKI, and help to improve clinical decision-making - such as earlier or delayed dialysis [4, 49], guiding decision-making, patient counseling and facilitating enrollee selection in interventional trials of AKI.

There were several limitations to our study. First, because of the limited sample size, the statistical significance may be improved after recruiting more patient samples. Second, only those patients undergoing cardiovascular surgery were included in this study and consequently other serious causes of AKI, such as sepsis or drug-induced AKI, are not represented. The results of our study could add to the value of AKI prediction after generalized cardiovascular surgery, and further large prospective studies are necessary to validate our results. Third, about $3 \%$ of urinary biomarker data were lost due to anuria at 12 and $24 \mathrm{~h}$ post-surgery. In addition, we did not obtain urine samples during surgery. Tubular injury may occur 30 min after commencing cardiopulmonary bypass in cardiac surgery with a peak immediately following completion of cardiopulmonary bypass [50]. However, our results clearly showed these markers had their peak level at $3 \mathrm{~h}$ after surgery and could predict advanced AKI. For clinical practice, urine collection after surgery was more feasible than collection during surgery. Finally, other urinary biomarkers, such as IL-18 and Lfatty acid binding protein, which also have promising results for predicting AKI, were not examined [51, 52].

Our study also has several strengths. First, this was a large multicenter prospective study and included a homogenous (post-cardiovascular surgery) patient population. Second, our study contained five biomarkers (including markers of proximal and distal tubular injury) and tested urine samples obtained in the ICU at $0,3,6$, 12 and $24 \mathrm{~h}$ after completion of surgery, which allowed exploration of the dynamic changes in their urinary concentrations and provided more comprehensive and credible results than previous studies [12, 53, 54]. Finally, this is, to our best knowledge, the first study using 


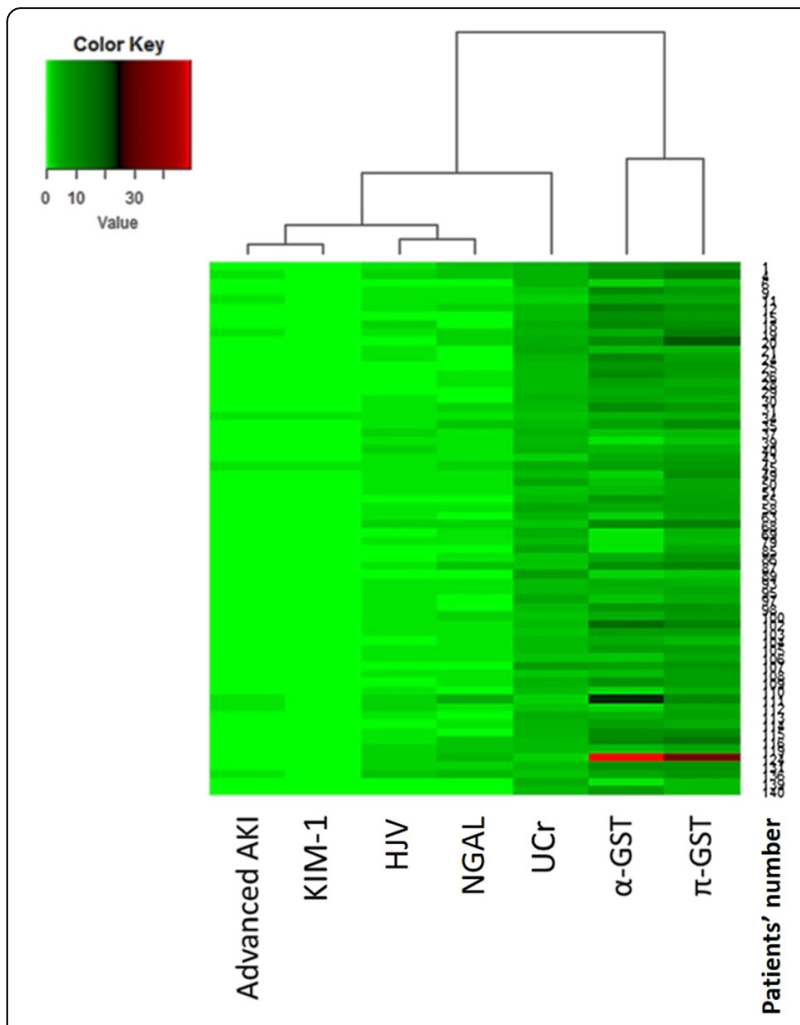

Fig. 3 Urinary biomarker concentrations related to advanced acute kidney injury (AKI). The creatinine-normalized biomarker concentrations were analyzed by unsupervised clustering to determine their relationship to advanced AKI. Full-length view of the cluster diagram has cases orientated along the vertical axis and biomarkers orientated along the horizontal axis. a-GST, a-glutathione S-transferase; $\pi$ GST, $\pi$-glutathione S-transferase; HJV, hemojuvelin; KIM-1, kidney injury molecule-1; NGAL, neutrophil gelatinase associated lipocalin; UCr, urinary creatinine

panels of urinary biomarkers in combination with clinical risk scores to focus on prediction of advanced AKI after cardiovascular surgery. Urinary biomarkers can lead to more accurate diagnosis of AKI and can further be used for recruitment of more homogenous patient populations when implementing a clinical trial [55].

\section{Conclusions}

In conclusion, our results indicate that creatininenormalized urinary biomarkers, particularly HJV and KIM-1 in combination, can improve the clinical predictive ability of Liano's score for advanced AKI and composite outcomes at an early time point after cardiovascular surgery. Risk prediction utilizing both biomarkers and clinical AKI score may enhance critical care and aid in forecasting the prognosis of postoperative patients. Larger prospective studies are necessary to confirm our observations and to validate the predictive panel for assessing clinical AKI.

\section{Additional file}

\begin{abstract}
Additional file 1: Supplementary methods: brief introduction of HJV, KIM-1, uNGAL, a-GST, ח-GST; Liano's score, National Taiwan University Study Group on Acute Renal Failure (NSARF) introduction and inotropic equivalents (IE). Table S1. Area under the receiver-operating characteristic curve (AUC) for normalized UHJV, UKIM-1 and serum creatinine for predicting advanced AKI at 3 and $6 \mathrm{~h}$ post-surgery. Table S2. Number and percent reclassified in advanced AKI prediction comparing initial model (Normalized [uHJV + uKIM-1]) to updated model (Liano's + Normalized [UHJV + UKIM-1]) at $3 \mathrm{~h}$ post-surgery. Table S3. Logistic regression analysis with variables available for predicting 90 days mortality after hospital discharge and composite outcomes. Figure S1. Receiver-operator characteristic curves for normalized UHJV, uKIM-1 and serum creatinine for predicting advanced AKI at 3 and $6 \mathrm{~h}$ post-surgery. Figure S2. Generalized additive models (GAM) plot for the probability of advanced AKI for normalized urinary HJV and KIM-1 at T3 (3 h post-surgery). (DOCX 723 kb)
\end{abstract}

\section{Abbreviations \\ AKI: Acute kidney injury; ANOVA: Analysis of variance; BMI: Body mass index; CABG: Coronary artery bypass graft; CCF ARF: Cleveland Clinic Foundation Acute Renal Failure; COPD: Chronic obstructive pulmonary disease; CPB: Cardiopulmonary bypass; eGFR: Estimated glomerular filtration rate; ELISA: Enzyme-linked immunosorbent assay; GAM: Generalized additive model; ICU: Intensive care unit; IDI: Integrated discrimination improvement; IL: Interleukin; kDa: KiloDalton; KDIGO: Kidney Disease: Improving Global Outcomes; MDRD: Modification of Diet in Renal Disease Study equation; NRI: Net reclassification improvement; SOFA: Sequential Organ Failure Assessment; uHJV: Urinary hemojuvelin; uKIM-1: Urinary kidney injury molecule-1; UNGAL: Urinary neutrophil gelatinase-associated lipocalin; ua- GST: Urinary a-glutathione S-transferase; uा-GST: Urinary $\pi$-glutathione S- transferase}

\section{Acknowledgements}

This study was supported by Taiwan National Science Council (101-2314-B002-085-MY3, 102-2314-B-002-140-MY2, 104-2314-B-002-125-MY3) and NTUH 106-FTN20/106-P02/UN106-014 106-S3582/105-P05/NN105-04/105-S3061/ VN104-07/104-S2718. This work was also supported by the Ministry of Science and Technology (MOST) of the Republic of China (Taiwan) (grant number, MOST 106-2321-B-182-002). We also express our sincere gratitude to all the staff of the Taiwan Clinical Trial Consortium, TCTC.

\section{Funding}

No funding was received for this analysis.

\section{Availability of data and materials}

The datasets used and/or analyzed during the current study are available from the corresponding author on reasonable request.

\section{Authors' contributions}

JJ contributed to data interpretation, drafting of the manuscript and critical revision of the manuscript. NH, TM and LW contributed to data collection and data interpretation and performed statistical analysis. RC contributed to interpretation and critical revision of the manuscript. WC and CC contributed to data collection and data interpretation. VC chaired the group, conceived and designed the study, performed statistical analysis and contributed to data collection, data interpretation, and critical revision of the manuscript. SC, JT, TS and KD contributed to interpretation and critical revision of the manuscript. All authors reviewed the manuscript. All authors read and approved the final manuscript.

\section{Ethics approval and consent to participate}

This study was approved by the research ethics review board of National Taiwan University Hospital (201105040RC) along with established written informed consent. This research was carried out in accordance with the approved guidelines. Written informed consent was obtained from all participants before inclusion.

\section{Competing interests}

The authors declare that they have no competing interests. 


\section{Publisher's Note}

Springer Nature remains neutral with regard to jurisdictional claims in published maps and institutional affiliations.

\section{Author details}

'Division of Nephrology, Department of Internal Medicine, Chi Mei Medical Center, Liouying, Tainan, Taiwan. ${ }^{2}$ NSARF group (National Taiwan University Hospital Study Group of ARF), Taipei, Taiwan. ${ }^{3}$ Department of Surgery, National Taiwan University Hospital, Taipei, Taiwan. ${ }^{4}$ Division of Nephrology, Department of Internal Medicine, National Taiwan University Hospital, Taipei, Taiwan. ${ }^{5}$ School of Biotechnology, Dublin City University, Glasnevin, Dublin 9, Ireland. ${ }^{6}$ Cleveland Clinic Lerner College of Medicine and Glickman Urological and Kidney Institute, Cleveland Clinic, Cleveland, USA. 'Division of Nephrology, Department of Internal Medicine, Chi-Mei Medical Center, Tainan, Taiwan. ${ }^{8}$ Department of Intensive Care Medicine, Chi Mei Medical Center, Liouying, Tainan, Taiwan. ${ }^{9}$ Chang Gung University College of Medicine, Taoyuan, Taiwan. ${ }^{10}$ Kidney Research Center, Department of Nephrology, Chang Gung Memorial Hospital, Taoyuan City, Taiwan.

\section{Received: 21 December 2017 Accepted: 9 April 2018}

Published online: 26 April 2018

\section{References}

1. Siew ED, Davenport A. The growth of acute kidney injury: a rising tide or just closer attention to detail? Kidney Int. 2015;87(1):46-61.

2. Lameire N, Van Biesen W, Vanholder R. Acute renal failure. Lancet (London, England). 2005;365(9457):417-30.

3. Chertow GM, Burdick E, Honour M, Bonventre JV, Bates DW. Acute kidney injury, mortality, length of stay, and costs in hospitalized patients. J Am Soc Nephrol. 2005;16(11):3365-70.

4. Zarbock A, Kellum JA, Schmidt C, Van Aken H, Wempe C, Pavenstadt H, Boanta A, Gerss J, Meersch M. Effect of early vs delayed initiation of renal replacement therapy on mortality in critically ill patients with acute kidney injury: the ELAIN randomized clinical trial. JAMA. 2016;315(20):2190-9.

5. Dennen P, Douglas IS, Anderson R. Acute kidney injury in the intensive care unit: an update and primer for the intensivist. Crit Care Med. 2010; 38(1):261-75.

6. Mishra J. Identification of neutrophil gelatinase-associated lipocalin as a novel early urinary biomarker for ischemic renal injury. J Am Soc Nephrol. 2003;14(10):2534-43.

7. Mishra J, Dent C, Tarabishi R, Mitsnefes MM, Ma Q, Kelly C, Ruff SM, Zahedi K, Shao M, Bean J, et al. Neutrophil gelatinase-associated lipocalin (NGAL) as a biomarker for acute renal injury after cardiac surgery. Lancet. 2005; 365(9466):1231-8

8. Young GH, Huang TM, Wu CH, Lai CF, Hou CC, Peng KY, Liang CJ, Lin SL, Chang SC, Tsai PR, et al. Hemojuvelin modulates iron stress during acute kidney injury: improved by furin inhibitor. Antioxid Redox Signal. 2014;20(8): 1181-94.

9. Siew ED, Ware LB, Ikizler TA. Biological markers of acute kidney injury. J Am Soc Nephrol. 2011;22(5):810-20.

10. Koyner JL, Garg AX, Coca SG, Sint K, Thiessen-Philbrook H, Patel UD, Shlipak MG, Parikh CR. Biomarkers predict progression of acute kidney injury after cardiac surgery. J Am Soc Nephrol. 2012;23(5):905-14.

11. Krawczeski CD, Goldstein SL, Woo JG, Wang Y, Piyaphanee N, Ma Q, Bennett M, Devarajan P. Temporal relationship and predictive value of urinary acute kidney injury biomarkers after pediatric cardiopulmonary bypass. J Am Coll Cardiol. 2011;58(22):2301-9.

12. Katagiri D, Doi K, Honda K, Negishi K, Fujita T, Hisagi M, Ono M, Matsubara $T$, Yahagi N, Iwagami M, et al. Combination of two urinary biomarkers predicts acute kidney injury after adult cardiac surgery. Ann Thorac Surg. 2012:93(2):577-83.

13. Hall IE, Coca SG, Perazella MA, Eko UU, Luciano RL, Peter PR, Han WK, Parikh CR. Risk of poor outcomes with novel and traditional biomarkers at clinical AKI diagnosis. Clin J Am Soc Nephrol. 2011;6(12):2740-9.

14. Arthur JM, Hill EG, Alge JL, Lewis EC, Neely BA, Janech MG, Tumlin JA, Chawla LS, Shaw AD. Evaluation of 32 urine biomarkers to predict the progression of acute kidney injury after cardiac surgery. Kidney Int. 2014; 85(2):431-8

15. Alge JL, Arthur JM. Biomarkers of AKl: a review of mechanistic relevance and potential therapeutic implications. Clin J Am Soc Nephrol. 2015;10(1):147-55.
16. Shaw M. The use of histologically defined specific biomarkers in drug development with special reference to the glutathione S-transferases. Cancer Biomark. 2005;1(1):69-74.

17. Srisawat N, Wen X, Lee M, Kong L, Elder M, Carter M, Unruh M, Finkel K, Vijayan A, Ramkumar $M$, et al. Urinary biomarkers and renal recovery in critically ill patients with renal support. Clin J Am Soc Nephrol. 2011:6(8):1815-23.

18. Ohnuma T, Uchino S, Toki N, Takeda K, Namba Y, Katayama S, Kawarazaki H, Yasuda H, Izawa J, Uji M, et al. External validation for acute kidney injury severity scores: a multicenter retrospective study in 14 Japanese ICUs. Am J Nephrol. 2015;42(1):57-64.

19. Varricatt VP, Rau NR, Attur RP, Baig WW. Validation of Liano score in acute renal failure: a prospective study in Indian patients. Clin Exp Nephrol. 2009; 13(1):33-7.

20. Thakar CV, Arrigain S, Worley S, Yared JP, Paganini EP. A clinical score to predict acute renal failure after cardiac surgery. J Am Soc Nephrol. 2005; 16(1):162-8.

21. Vincent JL, Moreno R, Takala J, Willatts S, De Mendonca A, Bruining $H_{\text {, }}$ Reinhart CK, Suter PM, Thijs LG. The SOFA (Sepsis-related Organ Failure Assessment) score to describe organ dysfunction/failure. On behalf of the Working Group on Sepsis-Related Problems of the European Society of Intensive Care Medicine. Intensive Care Med. 1996;22(7):707-10.

22. Wu VC, Ko WJ, Chang HW, Chen YS, Chen YW, Chen YM, Hu FC, Lin YH, Tsai $\mathrm{PR}$, Wu KD. Early renal replacement therapy in patients with postoperative acute liver failure associated with acute renal failure: effect on postoperative outcomes. J Am Coll Surg. 2007;205(2):266-76.

23. Wu VC, Ko WJ, Chang HW, Chen YW, Lin YF, Shiao CC, Chen YM, Chen YS, Tsai PR, Hu FC, et al. Risk factors of early redialysis after weaning from postoperative acute renal replacement therapy. Intensive Care Med. 2008; 34(1):101-8.

24. Shiao CC, Wu VC, Li WY, Lin YF, Hu FC, Young GH, Kuo CC, Kao TW, Huang DM, Chen YM, et al. Late initiation of renal replacement therapy is associated with worse outcomes in acute kidney injury after major abdominal surgery. Crit Care. 2009;13(5):R171.

25. Wu VC, Wang CH, Wang WJ, Lin YF, Hu FC, Chen YW, Chen YS, Wu MS, Lin YH, Kuo CC, et al. Sustained low-efficiency dialysis versus continuous venovenous hemofiltration for postsurgical acute renal failure. Am J Surg. 2010; 199(4):466-76.

26. Huang TM, Wu VC, Young GH, Lin YF, Shiao CC, Wu PC, Li WY, Yu HY, Hu FC, Lin JW, et al. Preoperative proteinuria predicts adverse renal outcomes after coronary artery bypass grafting. J Am Soc Nephrol. 2011;22(1):156-63.

27. Levey AS, Coresh J, et al. KDOQI clinical practice guidelines for chronic kidney disease: evaluation, classification, and stratification. Am J Kidney Dis. 2002;39(2 Suppl 1):S1-266

28. Wu VC, Huang TM, Shiao CC, Lai CF, Tsai PR, Wang WJ, Huang HY, Wang KC, Ko WJ, Wu KD, et al. The hemodynamic effects during sustained low-efficiency dialysis versus continuous veno-venous hemofiltration for uremic patients with brain hemorrhage: a crossover study. J Neurosurg. 2013;119(5):1288-95.

29. Young GH, Wu VC. KLOTHO methylation is linked to uremic toxins and chronic kidney disease. Kidney Int. 2012;81(7):611-2.

30. Wu VC, Wu CH, Huang TM, Wang CY, Lai CF, Shiao CC, Chang CH, Lin SL, Chen YY, Chen YM, et al. Long-term risk of coronary events after AKI. J Am Soc Nephrol. 2014;25(3):595-605.

31. Wu VC, Young GH, Huang PH, Lo SC, Wang KC, Sun CY, Liang CJ, Huang TM, Chen JH, Chang FC, et al. In acute kidney injury, indoxyl sulfate impairs human endothelial progenitor cells: modulation by statin. Angiogenesis. 2013;16(3):609-24

32. Palevsky PM, Liu KD, Brophy PD, Chawla LS, Parikh CR, Thakar CV, Tolwani AJ, Waikar SS, Weisbord SD. KDOQI US commentary on the 2012 KDIGO clinical practice guideline for acute kidney injury. Am J Kidney Dis. 2013; 61(5):649-72.

33. Friedman M. The use of ranks to avoid the assumption of normality implicit in the analysis of variance. J Am Stat Assoc. 1937;32(200):675-701.

34. Ralib AM, Pickering JW, Shaw GM, Devarajan P, Edelstein CL, Bonventre JV, Endre $\mathrm{ZH}$. Test characteristics of urinary biomarkers depend on quantitation method in acute kidney injury. J Am Soc Nephrol. 2012;23(2):322-33.

35. Hanley JA, McNeil BJ. The meaning and use of the area under a receiver operating characteristic (ROC) curve. Radiology. 1982;143(1):29-36.

36. Wu VC, Lo SC, Chen YL, Huang PH, Tsai CT, Liang CJ, Kuo CC, Kuo YS, Lee BC, Wu EL, et al. Endothelial progenitor cells in primary aldosteronism: a biomarker of severity for aldosterone vasculopathy and prognosis. J Clin Endocrinol Metab. 2011;96(10):3175-83. 
37. Shu KH, Wang CH, Wu CH, Huang TM, Wu PC, Lai CH, Tseng LJ, Tsai PR, Connolly R, Wu VC. Urinary pi-glutathione S-transferase predicts advanced acute kidney injury following cardiovascular surgery. Sci Rep. 2016;6:26335.

38. Hin LY, Lau TK, Rogers MS, Chang AM. Dichotomization of continuous measurements using generalized additive modelling-application in predicting intrapartum caesarean delivery. Stat Med. 1999;18(9):1101-10.

39. Steyerberg EW, Vickers AJ, Cook NR, Gerds T, Gonen M, Obuchowski N, Pencina MJ, Kattan MW. Assessing the performance of prediction models: a framework for traditional and novel measures. Epidemiology. 2010;21(1):128-38.

40. Eisen MB, Spellman PT, Brown PO, Botstein D. Cluster analysis and display of genome-wide expression patterns. Proc Natl Acad Sci U S A. 1998;95(25): 14863-8.

41. Devarajan P. Neutrophil gelatinase-associated lipocalin: a promising biomarker for human acute kidney injury. Biomark Med. 2010:4(2):265-80.

42. Lin L, Goldberg YP, Ganz T. Competitive regulation of hepcidin mRNA by soluble and cell-associated hemojuvelin. Blood. 2005;106(8):2884-9.

43. Shah SV, Baliga R, Rajapurkar M, Fonseca VA. Oxidants in chronic kidney disease. J Am Soc Nephrol. 2007;18(1):16-28.

44. Nath KA. Heme oxygenase-1: a provenance for cytoprotective pathways in the kidney and other tissues. Kidney Int. 2006;70(3):432-43.

45. Ichimura T, Bonventre JV, Bailly V, Wei H, Hession CA, Cate RL, Sanicola M. Kidney injury molecule-1 (KIM-1), a putative epithelial cell adhesion molecule containing a novel immunoglobulin domain, is up-regulated in renal cells after injury. J Biol Chem. 1998;273(7):4135-42.

46. Ichimura T, Asseldonk EJ, Humphreys BD, Gunaratnam L, Duffield JS, Bonventre JV. Kidney injury molecule-1 is a phosphatidylserine receptor that confers a phagocytic phenotype on epithelial cells. J Clin Invest. 2008;118(5): 1657-68.

47. Coca SG, Garg AX, Thiessen-Philbrook H, Koyner JL, Patel UD, Krumholz HM, Shlipak MG, Parikh CR. Urinary biomarkers of AKI and mortality 3 years after cardiac surgery. J Am Soc Nephrol. 2014;25(5):1063-71.

48. Endre ZH, Walker RJ, Pickering JW, Shaw GM, Frampton CM, Henderson SJ, Hutchison R, Mehrtens JE, Robinson JM, Schollum JB, et al. Early intervention with erythropoietin does not affect the outcome of acute kidney injury (the EARLYARF trial). Kidney Int. 2010;77(11):1020-30.

49. Gaudry S, Hajage D, Schortgen F, Martin-Lefevre L, Pons B, Boulet E, Boyer A, Chevrel G, Lerolle N, Carpentier D, et al. Initiation strategies for renal-replacement therapy in the intensive care unit. N Engl J Med. 2016;375(2):122-33.

50. Lannemyr L, Lundin E, Reinsfelt B, Bragadottir G, Redfors B, Oras J. Renal tubular injury during cardiopulmonary bypass as assessed by urinary release of N-acetyl-ss-D-glucosaminidase. Acta Anaesthesiol Scand. 2017;61(9):1075-83.

51. Wang J, Long Q, Zhang W, Chen N. Protective effects of exogenous interleukin 18-binding protein in a rat model of acute renal ischemiareperfusion injury. Shock. 2012;37(3):333-40.

52. Ferguson MA, Vaidya VS, Waikar SS, Collings FB, Sunderland KE, Gioules CJ, Bonventre JV. Urinary liver-type fatty acid-binding protein predicts adverse outcomes in acute kidney injury. Kidney Int. 2010;77(8):708-14.

53. Prowle JR, Calzavacca P, Licari E, Ligabo EV, Echeverri JE, Bagshaw SM, Haase-Fielitz A, Haase M, Ostland V, Noiri E, et al. Combination of biomarkers for diagnosis of acute kidney injury after cardiopulmonary bypass. Ren Fail. 2015;37(3):408-16.

54. Yang $\mathrm{CH}$, Chang $\mathrm{CH}$, Chen TH, Fan PC, Chang SW, Chen CC, Chu PH, Chen YT, Yang HY, Yang CW, et al. Combination of urinary biomarkers improves early detection of acute kidney injury in patients with heart failure. Circ J. 2016;80(4):1017-23.

55. Parikh CR, Moledina DG, Coca SG, Thiessen-Philbrook HR, Garg AX. Application of new acute kidney injury biomarkers in human randomized controlled trials. Kidney Int. 2016;89(6):1372-9.

Ready to submit your research? Choose BMC and benefit from:

- fast, convenient online submission

- thorough peer review by experienced researchers in your field

- rapid publication on acceptance

- support for research data, including large and complex data types

- gold Open Access which fosters wider collaboration and increased citations

- maximum visibility for your research: over $100 \mathrm{M}$ website views per year

At BMC, research is always in progress.

Learn more biomedcentral.com/submissions 